\section{Salud de los Pueblos Indígenas de las Américas ${ }^{1}$}

\section{ANTECEDENTES}

Como es de conocimiento general, el estado de salud de las comunidades indígenas de las Américas y la cobertura de los servicios de salud que reciben reflejan graves desigualdades en relación con el resto de la población. Si bien no se cuenta con datos epidemiológicos nacionales igualmente fiables sobre el estado de salud y enfermedad de los más de 43 millones de personas indígenas de la Región, diversos estudios muestran lo siguiente:

- En los lactantes indígenas de Panamá, la mortalidad es 3,5 veces más alta que en otros niños (1).

- En Bolivia, 20\% de los lactantes indígenas mueren antes de cumplir el primer año de vida y $14 \%$ de los supervivientes mueren antes de llegar a la edad escolar (2).

- La mortalidad por diabetes entre los indígenas de los Estados Unidos de América es 166\% más elevada que en la población en general (3).

- En el Canadá, la tasa estandarizada según la edad de muertes por accidentes y violencia correspondiente a la población indígena es de 81 por 100000 habitantes, comparada con 46 por 100000 en el resto de la población (4).

- Los niños mayas de edad escolar en Belice mostraron el mayor retraso de crecimiento de todos los grupos étnicos (5).

- En Honduras, la esperanza de vida es de 36 años en hombres indígenas frente a 65 en los hombres en general, y de 43 años en mujeres indígenas frente a 70 en toda la población femenina del país (6).

- En México, la mortalidad entre los niños indígenas en edad preescolar es de $12,8 \%$ frente a solo $4,8 \%$ a nivel nacional (7).

- En Guatemala, en 1994 la tasa de mortalidad materna en la población indígena era $83 \%$ más alta que la tasa nacional (8).

Los países de la Región están buscando nuevas formas de enfocar la prestación de los servicios de salud y soluciones innovadoras para problemas de salud que permitan satisfacer las necesidades de estas poblaciones vulnerables. El reto que constituye hacerles frente a problemas ya reconocidos, como los que se acaban de mencionar, se ve agravado por el hecho de que en la Región hay más de 
400 distintos grupos étnicos con una rica diversidad de idiomas, costumbres y creencias. Esta heterogeneidad excluye la posibilidad de usar los mismos enfoques e intervenciones en todas partes. En 1992 el Subcomité de Planificación y Programación del Comité Ejecutivo de la OPS propuso que la Organización considerara detenidamente la salud y el bienestar de los pueblos indígenas de las Américas. Seguidamente, en 1993, nombrado por las Naciones Unidas "Año de los Pueblos Indígenas", La OPS emprendió una iniciativa en la que se solicitó la colaboración de los pueblos indígenas para planificar una respuesta. Se realizó un taller de consulta en Winnipeg, Canadá, en el que participaron representantes de los pueblos indígenas, gobiernos y otros grupos de 18 países. Las recomendaciones emanadas de ese encuentro se consolidaron en la iniciativa Salud de los Pueblos Indígenas de las Américas, propuesta que se presentó a los Cuerpos Directivos de la OPS y que fue aprobada mediante la Resolución CD37.R5 (anexo) en septiembre de 1993.

Sobre la base de las recomendaciones de Winnipeg se establecieron cinco principios que guían el trabajo de la Organización con las comunidades indígenas, proporcionan criterios de monitoreo y servirán como fundamento para una evaluación al término del Decenio Internacional de las Poblaciones Indígenas del Mundo, en 2004. Esos principios son que la salud de los pueblos indígenas requiere un enfoque integral y que estos pueblos tienen, además, derecho a la autodeterminación, a la participación sistemática, a que se respeten y revitalicen sus culturas, y a la reciprocidad en las relaciones con otros grupos.

En 1994, los participantes de dos talleres subregionales en Santa Cruz, Bolivia, y Quetzaltenango, Guatemala, ratificaron los principios acordados en Winnipeg y las metas presentadas en la Resolución CD37.R5. Para 1995, se había establecido un Plan de Acción de manera que la voluntad política expresada por los Estados Miembros se plasmara en acciones concretas y sostenibles.

Este asunto ha empezado también a recibir la atención que merece en el ámbito mundial. En 1996, la 49. ${ }^{a}$ Asamblea Mundial de la Salud aprobó una resolución sobre la ejecución del Decenio Internacional de las Poblaciones Indígenas del Mundo (1994-2004) y el Grupo de Trabajo sobre las Poblaciones Indígenas de la Comisión de Derechos Humanos de las Naciones Unidas incluyó la salud en su programa de temas. En ambas ocasiones se reconoció el trabajo en curso en la Región de las Américas por haber estimulado una toma de conciencia en lo que se refiere a las desigualdades en el estado de salud y al acceso a los servicios de las comunidades indígenas.

\section{ADELANTOS LOGRADOS}

Hasta 1996 el trabajo de la iniciativa se concentró en las cinco áreas siguientes: fortalecimiento de la capacidad operativa y desarrollo de alianzas; colaboración con los Estados Miembros para poner en marcha los procesos y proyectos nacionales y locales; adelanto de proyectos en áreas programáticas prioritarias; fortalecimiento de los sistemas de salud tradicionales, y diseminación de información científica, técnica y pública.

Fortalecimiento de la capacidad y las alianzas. Esta área de trabajo constituyó el foco principal de la iniciativa antes de elaborarse el Plan de Acción en 1995 y sigue siendo importante en la actualidad, ya que el Plan se está modificando de acuerdo con las experiencias de los tres primeros años. Los talleres subregionales proporcionaron capacitación de personal y en las iniciativas de promoción se identificó en las oficinas de la OPS, los ministerios de salud y las organizaciones indígenas a las personas clave que serían las responsables de llevar a cabo la Resolución CD37.R5. La OPS ha formado alianzas con organizaciones de grupos indígenas, bancos de desarrollo, organismos de las Naciones Unidas e instituciones nacionales con el fin de trabajar en áreas de interés en común.

Apoyo a los Estados Miembros. La OPS proporciona apoyo a los países en la formulación de planes nacionales y locales, políticas y procesos destinados a beneficiar a los pueblos indígenas. Este trabajo se está realizando en fases, otorgando prioridad a aquellos países que tienen grandes poblaciones indígenas, ya sea en números absolutos o en proporción de la población total. No obstante, todos los países se benefician de las actividades subregionales y regionales.

Desde el principio se ha insistido en la participación de representantes indígenas y especialmente de mujeres. El trabajo en los países se ha facilitado gracias al esfuerzo conjunto de esos representantes, así como el de las Oficinas de Representación de la OPS en los países y el del personal complementario designado en algunos de los ministerios de salud. Los ministerios de salud y representantes de la OPS han preparado informes de los logros alcanzados en 18 países y estos formarán la base de los resúmenes que se publicarán sobre el trabajo presente y futuro.

Adelanto de proyectos prioritarios. Esta tercera área de trabajo comprende el diseño de proyectos 
relacionados con problemas de salud prioritarios y poblaciones vulnerables y también la movilización de recursos para realizarlos. Basándose en una consulta permanente con las comunidades indígenas, la iniciativa ha promovido proyectos y actividades en varias áreas programáticas. El mayor progreso se ha logrado en relación con el abastecimiento de agua y el saneamiento básico, la salud de la mujer indígena, la salud mental, la prevención del cólera, las enfermedades prevenibles por vacunación y el compromiso de organizaciones no gubernamentales con el mejoramiento de la salud. Actualmente hay 18 programas regionales para los cuales hay propuestas, actividades concretas de cooperación técnica, planes teóricos y proyectos extrapresupuestarios en diferentes etapas de desarrollo.

Fortalecimiento de los sistemas de salud tradicionales. Un aspecto importante de este trabajo es crear una articulación más estrecha entre el sistema de salud indígena, que se caracteriza por una multiplicidad de prácticas y practicantes, y el sistema oficial que brindan los gobiernos. Es probable que la mayoría de los 43 millones de personas indígenas en las Américas en realidad no tenga acceso a los servicios básicos de salud primaria que ofrecen los programas patrocinados por los gobiernos, ya que aun cuando esos servicios se encuentran físicamente accesibles, suele haber obstáculos de índole financiera, geográfica o cultural que entorpecen su uso. Frente a esas barreras, las comunidades dependen de los terapeutas tradicionales y espirituales para promover la salud y prevenir y tratar las enfermedades comunes. En varios proyectos entre países los terapeutas tradicionales han tenido la oportunidad de reunirse para intercambiar conocimientos. En algunos países se han establecido organizaciones no gubernamentales (ONG) de terapeutas tradicionales para lograr un mayor reconocimiento nacional y servicios de salud de mejor calidad. Es posible que se necesiten medidas legislativas para evitar que los terapeutas tradicionales encuentren obstáculos al practicar su oficio o que se les niegue acceso a los productos que acostumbran usar. Algunos grupos tienen como objetivo restituir los conocimientos perdidos debido a la devaluación de las prácticas tradicionales y a la falta de interés de los jóvenes en aprenderlas. Además, es preciso educar a los trabajadores de salud que prestan servicios en las comunidades multiculturales para que estén al tanto de las prácticas de salud indígenas.

Promoción del intercambio de información y la investigación. La quinta área de trabajo se refiere a la búsqueda y el desarrollo de mecanismos eficientes para coordinar, promover, diseminar y compartir información científica y técnica. Hasta fines de 1996 se habían preparado y distribuido 23 documentos y publicaciones y en abril de 1997 se estaban elaborando ocho más. En la Región circulan un folleto y un video en inglés y español acerca de la iniciativa. En 1995 se reunió un grupo de trabajo para iniciar la asignación de prioridades de investigación y formular proyectos colaborativos en ese campo. Hubo un intercambio de experiencias y puntos de vista entre tres investigadores indígenas que forman parte de una red de científicos cuya misión es proporcionar orientación para tratar las numerosas cuestiones éticas inherentes a la investigación.

Desde el principio de la Iniciativa, la OPS ha hecho hincapié en el enfoque intersectorial y en la necesidad de trabajar en colaboración con otras entidades para llevar a cabo el Plan de Acción u otros planes complementarios. En mayo de 1996, el Director de la OPS firmó un acuerdo con el Parlamento Indígena de las Américas. Este estipula que la OPS trabajará con otros parlamentos internacionales para desarrollar y ejecutar un plan que ayude a los países a preparar una agenda legislativa para las políticas de salud y a producir leyes que protejan a los terapeutas tradicionales y a las plantas medicinales y que estimulen a los legisladores a emprender otras acciones a favor de la salud de los pueblos indígenas.

Lecciones aprendidas y retos por encarar. Se han aprendido lecciones importantes durante la ejecución de la Resolución CD37.R5 y la iniciativa. Estas lecciones proveerán los criterios para reorientar el trabajo futuro.

La movilización de recursos ha tomado más tiempo del anticipado y se están llevando a cabo negociaciones con donantes. En el futuro, la OPS se centrará en actividades que se puedan realizar con recursos limitados, animará a los programas y a los países a que asignen a ellas pequeñas partidas de sus presupuestos ordinarios y tratará de encontrar donantes menos tradicionales.

El seguimiento de los componentes interprogramáticos de la iniciativa ha sido un desafío, especialmente cuando un proyecto general incluye actividades relacionadas con la salud indígena. Se está buscando una forma mejor de llevar un control de las actividades realizadas en los distintos programas.

Pocos países recolectan y analizan estadísticas vitales o sobre los servicios de salud según el origen étnico de los usuarios, así que ha sido difícil establecer buenos datos de referencia o evaluar adecuadamente la salud y las condiciones de vida de los pue- 
blos indígenas de la Región. En el futuro se hará un esfuerzo por promover la desagregación de datos por grupos étnicos con el fin de detectar y observar las desigualdades del estado de salud y del acceso a los servicios. Si eso no es posible a corto plazo, habrá que usar otros indicadores, por ejemplo, los datos de municipalidades con un mínimo de 50\% de habitantes indígenas y compararlos con los datos nacionales.

No se ha avanzado lo suficiente con respecto a la participación sistemática de las personas indígenas y sus organizaciones. Muy pocos países han establecido comisiones gubernamentales técnicas con representantes indígenas, si bien algunos han formado grupos de estudio interprogramáticos y otros, comisiones en los ministerios de salud.

Los institutos nacionales indigenistas no siempre cuentan con la dirección de personas indígenas $\mathrm{y}$, cuando la tienen, no asignan una gran prioridad a la salud.

\section{PLANES PARA EL FUTURO}

De acuerdo con la experiencia adquirida de 1993 en adelante, se han propuesto para 1997-1998 las siguientes cuatro áreas principales de trabajo:

Planificación estratégica y gerencia. Esta primera línea de trabajo es un elemento crítico para que la iniciativa pueda adherirse a los principios enunciados en la Resolución CD37.R5. Los países han respondido a la iniciativa con diversos grados del entusiasmo y los informes de progreso que han presentado hasta 1996 reflejan una gama muy variada de esfuerzos. Se espera que la iniciativa pase por cuatro etapas en los países: promoción, iniciación, consolidación y evaluación.

Con objeto de movilizar recursos complementarios de apoyo a las primeras actividades de iniciación, se ha presentado al Banco Interamericano de Desarrollo (BID) y a otros donantes un proyecto titulado "Hacia la salud de los pueblos indígenas: procesos y proyectos". Parte del proyecto está dirigido a fomentar la capacitación de líderes indígenas y la formación de redes de trabajo subregionales organizadas por ellos.

Programas de prioridad. La OPS continuará con los programas de prioridad ya establecidos y añadirá nuevos campos de acción a medida que los países muestren interés en emprender proyectos vinculados con las comunidades indígenas. Será de suma importancia crear un banco de datos sobre recursos humanos que incluya una lista de expertos indígenas idóneos para cada programa prioritario. En los lugares donde haga falta personal con conocimientos especializados, se promoverá la formación de profesionales indígenas y los países podrán reservar becas de la OPS para encauzar su capacitación hacia áreas de prioridad. También se estimulará la incorporación de componentes de salud indígena en proyectos de investigación de más amplio espectro y se movilizarán recursos para esos fines. Este enfoque colaborativo ya ha empezado a dar frutos.

Organización y prestación de servicios de salud en comunidades multiculturales. Estas actividades se centrarán en adiestrar a trabajadores de salud para que proporcionen atención compenetrada con la cultura, y en elaborar y poner a prueba en el terreno normas y materiales didácticos complementarios. En colaboración con el Parlamento Indígena y otros parlamentos internacionales, se formularán y se difundirán pautas para reglamentar, legitimar y legalizar la práctica de los terapeutas tradicionales. Dado que muchos grupos indígenas están situados en zonas escasamente pobladas, se buscarán, sistematizarán, documentarán y diseminarán medios innovadores de proporcionarles acceso a servicios básicos de salud pública y clínicos, mediante la cooperación técnica entre varios países.

Producción y diseminación de información pública científica y técnica. En 1997 y 1998 se trabajará para divulgar información sobre la salud y las condiciones de vida de pueblos indígenas en diversos países, subregiones y la Región en general. Se brindará cooperación a los países para que preparen y publiquen documentos sobre la salud de los pueblos indígenas y se difundirán asimismo los informes de trabajo y documentos programáticos de los programas prioritarios. La OPS está explorando las posibles fuentes de financiamiento y apoyo técnico para una serie de videos sobre la cultura y el desarrollo de la salud de los pueblos indígenas. En los videos se hará hincapié en los importantes aportes de las culturas indígenas a la salud y en la variedad de métodos novedosos que se están desarrollando como parte de la iniciativa.

Trabajan junto con la OPS en la iniciativa otros organismos de las Naciones Unidas y la Organización de los Estados Americanos. Esas alianzas serán importantes en la movilización de recursos financieros y técnicos en los niveles nacional y regional. Se buscará también el concurso de otras 
instituciones que tengan experiencia en materia de salud indígena.

En su 40. ${ }^{a}$ Reunión, el Consejo Directivo de la OPS reiteró su compromiso con las metas del Decenio internacional de las Poblaciones Indígenas del Mundo y aprobó las actividades propuestas en la iniciativa para el período de 1997-1998 mediante la Resolución CD40.R6. Encareció también a los Estados Miembros a poner empeño en descubrir, vigilar y rectificar las desigualdades en el estado de salud y en los servicios básicos de salud de los grupos vulnerables, incluidas las poblaciones indígenas.

La equidad es el fundamento del trabajo de la OPS en el terreno de la salud pública. Mientras algunas comunidades tengan menos servicios, una mayor carga de morbilidad y menos oportunidades que otras, no podrá materializarse la meta de salud para todos. Solo el esfuerzo persistente a favor de las comunidades indígenas de las Américas hará que los países puedan compartir y celebrar su éxito al término del Decenio Internacional de las Poblaciones Indígenas del Mundo.

\section{SYNOPSIS}

\section{Health of Indigenous Peoples}

The Health of Indigenous Peoples Initiative was launched by PAHO in 1993 in response to recommendations issued by a workshop in Winnipeg, Canada. Its purpose was to address the inequality that exists in the Americas in the health of indigenous populations and their access to health care. Since 1993 the initiative has focused on the five following lines of action: building capacity and alliances; developing national and local processes and projects; establishing projects dealing with priority health problems; strengthening traditional health systems, and disseminating scientific, technical, and health information. A multistage approach has been used to introduce the initiative within the countries, and different results have been observed. The current challenges involve mobilizing extrabudgetary funds, coordinating the activities of different programs and agencies, and assuring the sustained representation of indigenous peoples at the regional and national levels. This report looks at the progress attained so far and describes future initiatives.

\section{REFERENCIAS}

1. Amaris A, Flores C, Mojica J. Mortalidad infantil en Panamá: un análisis de sus tendencias derivadas del censo 1990. Panamá, Panamá; 1992.

2. Centro de Comunicación y Desarrollo Andino. Los jampiris de Ragaypampa. Bolivia: Cenda; 1993.

3. United States Indian Health Service. Trends in Indian health 1996. Washington, DC: Department of Health and Human Services, USIHS; 1996.
4. Canada, Ministry of National Health and Welfare, Ministry of Supply and Services. Aboriginal health in Canada: Ottawa: MSS; 1992.

5. Belize, Ministry of Education; Pan American Health Organization, Institute of Nutrition of Central America and Panama; United Nations Children's Fund; United Nations Development Programme. Closing the gap: national height census of schoolchildren in Belize,
1966. Belmopan: Ministry of Education; 1996.

6. Rivas R. Pueblos indígenas y garífunas. Tegucigalpa, Honduras: Guaymuras; 1993.

7. México, Secretaría de Salud, Instituto Nacional Indigenista. La salud de los pueblos indígenas en México. México. DF: Secretaría de Salud; 1993.

8. Velázquez L. Mujer maya y salud. Ciudad de Guatemala: 1994

\section{ANEXO}

\section{Resolución CD37.R5, Salud de los Pueblos Indígenas de las Américas}

\section{La XXXVII Reunión del Consejo Directivo,}

Visto el documento CD37/20 sobre la iniciativa Salud de los Pueblos Indígenas de las Américas;

Tomando en cuenta las recomendaciones efectuadas por los participantes en la Reunión de Trabajo sobre Pueblos Indígenas y Salud, realizada en la ciudad de Winnipeg, Manitoba, Canadá, del 13 al 17 de abril de 1993;

(continúa en la página siguiente) 
Reconociendo que las condiciones de vida y de salud que prevalecen entre una población estimada en unos 43 millones de indígenas en la Región de las Américas son deficitarias, expresándose en una mortalidad excesiva por causas evitables y en una menor esperanza de vida al nacer, lo cual demuestra la persistencia y aun la acentuación de las desigualdades de las poblaciones indígenas en relación con otros grupos sociales homólogos;

Considerando las aspiraciones de los pueblos indígenas de asumir el control de sus propias instituciones y formas de vida, la necesidad de fortalecer su propia identidad, así como de que se respeten sus derechos en cuanto a la salud y al medio ambiente;

Reconociendo la particular contribución de los pueblos indígenas al mantenimiento de la diversidad étnica y cultural de las Américas, a la biodiversidad y al equilibrio ecológico, y muy especialmente a la salud y nutrición de la sociedad;

Resaltando la necesidad de revalorar y respetar la integridad de valores y prácticas sociales, culturales, religiosos y espirituales propios de los pueblos indígenas, incluidos aquellos que tienen relación con la promoción y el mantenimiento de la salud, y con el tratamiento de las enfermedades y dolencias, y

Reiterando la importancia de la estrategia de transformación de los sistemas nacionales de salud y de la propuesta de desarrollo de modelos alternativos de atención a nivel de los sistemas locales de salud (SILOS) como un recurso táctico valioso y requisito fundamental para la superación de los actuales problemas de déficit de cobertura, falta de acceso y baja aceptabilidad de los servicios de salud entre las poblaciones indígenas,

\section{Resuelve:}

1. Adoptar el documento CD37/20, que describe la iniciativa Salud de los Pueblos Indígenas de las Américas y el informe de la Reunión de Trabajo de Winnipeg con las conclusiones y recomendaciones sobre las que se basa la iniciativa.

2. Instar a los Gobiernos miembros a que:

a) Promuevan el establecimiento o fortalecimiento de una comisión técnica de alto nivel u otro mecanismo de concertación que se considere apropiado, con participación de líderes y representantes de pueblos indígenas, para la formulación de políticas y estrategias, y el desarrollo de actividades de salud y medio ambiente dirigidas hacia poblaciones indígenas específicas;

b) Fortalezcan la capacidad técnica, administrativa y gerencial de las instituciones nacionales y locales responsables de la salud de las poblaciones indígenas, a fin de superar progresivamente la falta de información en este campo y asegurar mayor acceso a servicios de salud y atención de calidad, contribuyendo así a mejores niveles de equidad;

c) Pongan en marcha las acciones intersectoriales que corresponda en los campos de la salud y el medio ambiente tanto a nivel del sector oficial como a través de organizaciones del sector no gubernamental (ONG), universidades y centros de investigación que trabajan en colaboración con organizaciones indígenas;

d) Promuevan la transformación de los sistemas de salud y apoyen el desarrollo de modelos alternativos de atención de la población indígena, dentro de la estrategia de los SILOS, incluyendo la medicina tradicional y la investigación sobre su calidad y seguridad; 
e) Promuevan el desarrollo de programas de prevención de enfermedades y promoción de la salud para atender problemas y áreas de mayor importancia en materia de salud indígena en sus países.

3. Solicitar al Director, dentro de la disponibilidad de recursos, que:

a) Promueva la participación de los indígenas y sus comunidades en todos los aspectos del trabajo de la Organización sobre salud de los pueblos indígenas;

b) Identifique, dentro de los programas de cooperación, recursos de cooperación técnica y preste apoyo a la movilización de recursos adicionales a nivel internacional y nacional para la puesta en marcha y evaluación de la iniciativa Salud de los Pueblos Indígenas de las Américas;

c) Coordine el esfuerzo regional para promover la formación de redes de información y colaboración recíproca entre organizaciones, centros e instituciones que trabajan en el campo de la salud de pueblos, organizaciones y comunidades indígenas, utilizando los mecanismos, iniciativas y programas de la Organización ya existentes en la Región y en los países, y procurando obtener la cooperación de otros organismos y organizaciones;

d) Amplíe las actividades de evaluación de las condiciones de vida y situación de salud para incluir a los pueblos indígenas de la Región, con el fin de superar paulatinamente la actual falta de información en este campo tanto a nivel regional como a nivel de los países;

e) Promueva la investigación colaborativa a nivel de la Región y países seleccionados en temas prioritarios de salud y atención de salud de los pueblos indígenas. 